\title{
Applying the Time and Space Forms of Poetry to Creative Design
}

\author{
Mo-Li Yeh ${ }^{1}$, Ming-shean Wang ${ }^{2}$, and Pey-Chwen Lin $^{3}$ \\ ${ }^{1}$ Design College, Hsing Wu University \\ Linkou, Taipei 24452, Taiwan \\ ${ }^{2}$ Digital and Design Department, Mingdao University \\ Peetow, Changhua 52345, Taiwan \\ ${ }^{3}$ New Media Art, National Taiwan University of Art \\ Ban Ciao City, Taipei 22058, Taiwan \\ 1101moli@gmail.com
}

\begin{abstract}
In recent years, countries worldwide have actively employed culture-inspired creativity to add value to designs. Poetry is a means by which poets express profound and subtle emotions and thoughts using refined words. Classical and renowned poems that have survived for thousands of years are undoubtedly excellently refined masterpieces. We established a series of operable steps for converting abstract creative poetry concepts into concrete poetry designs to enhance the enriched literary connotations of designs and improve the values of the affective and aesthetic experience of poetry. Based on the spiritual characteristics of classical Chinese poetry and theories related to time and space, this study references literature regarding the feasibility of applying cultural creativity to designs and summarizes 4 forms of poetry, namely, time/space reflection, time/space separation, time/space sympathy, and time/space integration. When designing actual cases, we considered the method by which a logical inference procedure can be developed and the concrete steps for reasonably converting poetry into design. Subsequently, this study describes the extraction and control of critical meanings from the context and themes of traditional poetry to analyze structural forms and content of time and space. The converted poetry design cases were then integrated to construct a creative design application model for the time and space forms of poetry. Integrating traditional poetry culture with modern designs provides the current cultural and creative industry with a feasible design model. Furthermore, creative design revitalizes traditional poetry, which enables the value of poetry to be fully developed and realized in cultural and creative applications.
\end{abstract}

Keywords: poetry, time and space, cultural creativity, creative design.

\section{Introduction}

Various countries have attempted to leverage their cultural characteristics to enhance the implied value of creative design and promote a culturally oriented aesthetic economy. According to Lin (2007), culture is a lifestyle; design is a taste; creativity is a 
type of recognition achieved through affection; and industry is a medium, approach, or method that can be used to realize creativity in cultural design. The essence of creative cultural design is extracting cultural elements, converting cultural symbols, and endowing designs with novel aesthetic implications. Most cultural product designs currently available in Taiwan are derived from tangible cultural assets such as historical sites and buildings, natural scenery, cultural landscapes, and utensils; however, few creative ideas have originated from intangible cultural assets. Poetry is a root of traditional culture, providing an explicit form of communication and implicit representation and connotations that can be used to inspire creative cultural design. Thus, the intangible cultural elements of poetry can be extracted and referenced to conceptualize creative cultural design. We explored converting the time and space forms of classical poetry and the feasibility of applying these forms to modern design. This application should benefit cultural preservation, enhancing the meaning and profoundness of overall creative cultural design.

\section{Relationship between Poetry and Design}

\subsection{Poetry: Design Thinking of Cultural Creativity}

Li (1996) defined culture as "products created by people through common activities; culture includes the tools used by people, the codes and systems on which social life is based, art products that enrich people's spiritual life, and people's mental activities during creative processes." Chinese history and culture spans 5,000 years, and the culture of classical poetry that has accumulated for generations is an invaluable and intangible cultural asset. The German philosopher Cassirer (1944) stated that culture is the externalization and objectification of human beings and the actualization and concretization of symbolic activities. In addition, all human cultural forms are symbolic forms. Influenced by this theory, Langer (1953), an American philosopher, argued, "Art is the creation of forms symbolic of human feeling." This argument profoundly influenced modern aesthetic theories. Poetry culture comprises the artistic expressions of concrete text symbols that poets use to express psychological and emotional dimensions. In his book Abstraction and Empathy, the German philosopher Worringer (2007) indicated that humans derive happiness from art primarily because people can extract individual objects and events of the external world from all contingency and caprice; thus, abstract forms are used to eternalize the extracted objects. Regarding the creation of poetry, Zhu (1988) asserted that depression originates from discontentment with the finiteness or limitations of life and that imagination and fantasies represent the pursuit of the infinite. In addition, he claimed that "poetry consists of implicit and explicit meanings. Implicit meanings focus on the principles and reasons of objects, whereas explicit meanings emphasize the appearance. Poetry is only complete when it contains both implicit and explicit meanings." Put simply, poetry is the process of using finite language to seek infinite imagination and create poetic images that stand the test of logical thinking. Rapaille (2006) reasoned that effective commodities must be able to activate the cultural codes relevant to consumers. Thus, the classical poetry culture that has been transmitted through the generations can be 
employed to interpret the implicit forms of poetic emotion. These interpretations can be converted to poetic products that feature cultural creativity, satisfying the desires and demands of contemporary people, who experience internal emptiness, and enhancing the meaning and depths of creative cultural design. The inner cultivation of poets often affects the level of artistic conception and emotions delivered through their works. In the Shanjingju Hualun (Shanjingju Critiques of Paintings), the Qing Dynasty artist Fang Xun stated that "the magic of brushes and ink lies in the magic of the painters' ideas. Therefore, ancient painters take up brushes to paint only after they have developed ideas." He also stated that "painters must first conceptualize ideas to position their works. Surprising ideas result in interesting paintings; advanced ideas yield thought-provoking paintings; pioneering ideas create forward-looking paintings; profound ideas constitute deep paintings; nostalgic ideas form reminiscent paintings; ordinary ideas give birth to mediocre paintings; and vulgar ideas generate inferior paintings." These comments enforce the viewpoint that the depth of poetic emotions and ideas determines the elegance of the ideologies underlying poetic works. Famous poems that have survived thousands of years are typically works that have undergone constant revisions. Consequently, poetry can be used to enhance the literary connotations of designs, endowing them with poetic conceptions and aesthetic experiences.

\subsection{Significance of the Time and Space Design in Poetry}

In his book Chinese Poetics: Design, Huang (1976) adopted the perspective of modern aesthetics and established an objective aesthetic system in abstract poetic art, implementing interdisciplinary integration. Specifically, art psychology and the relations among various art forms (e.g., plastic arts) were emphasized to develop the theoretical core of poetics. This facilitated exploring the artistic qualities of classical Chinese poetry, allowing the thoughtfulness in these poems to be acknowledged and understood. Li (2007) argued that "the conceptualization of poems is the most creative and crucial stage in creating poetry. An excellent poem is typically based on excellent ideas, which are essentially creative imagination. This imagination is an expression of creative association." Li (2007) further asserted that "time and space have mystical and undeniable bonds with human beings, who are integral components of the universe. Time and space are also eternally linked to poetry, which is a wonderful art form that never withers. Only in the contexts of time and space can poetry blossom, radiate fragrance, and glow with its unique hues." In his sonnets, Shakespeare expressed his ideas as a poet and author, regarding the transience of time and the eternity of poetry. Huang (1976) offered a poetic definition of poetry: "The sayings that poetry is an audible landscape and painting in time, or that poetry is visible music and painted time are both reasonable because poetry is an art interwoven in time and space. Rather than static, poetry is extensive and variable through time and space." Therefore, time and space design in poetry is vital when examining Chinese poetry because the contents of poems are primarily based on time, space, emotion, and reason. These complex elements connect and transcend time and space, becoming alternately evident or absent during the natural transition of time and space. The magical and seamless immersion between human beings and time and space, and the connection between emotions and 
philosophies cannot be uprooted from the contexts of time and space. Instead, the interactions of concrete temporal and spatial images are essential for visualizing these relationships. Therefore, time and space design is the most vital link in Chinese poetry. In summary, time and space has been explored by domestic and international philosophers and scientists in ancient and modern times; it is a vital aesthetic theme expressed by domestic and international poets in ancient and contemporary times.

\subsection{Applying the Time and Space Forms of Poetry to Design}

The time and space forms of art works are objectified and concretized expressions of the artists' aesthetic awareness of time and space realized using artistic approaches. Time and space are two basic forms through which artistic images sustain and express themselves (Li, 2007). Xie He was a scholar in the Southern Qi period who proposed the idea of six principles in the Guhua Pinlu (A Critique of Ancient Paintings). Specifically, the concept of operating position essentially refers to the temporal and spatial structures of works. The composition principles used in contemporary painting theories are derived from the classical concept of operating position, and spatial combination is a critical aesthetic principle for determining the formal beauty in contemporary paintings. In his book Aesthetics: Pursuits of Torment, Xia Fang defined formal beauty as the natural attributes (e.g., color, form, and sound) that constitute the material appearance of objects, combined with the aesthetic characteristics generated using artistic rules (e.g., symmetry, balance, proportion, and repetition). This definition is consistent with those stated in numerous design principles. In addition, the temporal and spatial structures of poetry vary substantially and constantly, resembling changes in clouds that spread across an autumn sky. Consequently, these structures cannot be regulated using fixed models. Analyses of structural forms vary depending on the perspective adopted. In his book, Huang (1976) conducted a detailed analysis of temporal and spatial art in poetry. In the current study, we adopted the poetry classification that Huang proposed for time and space design, using his examples to explain particular poems; thus, the correlations between these principles and those of art design were identified. Chou (2002) examined the mental processes that artists experience during creation, stating the following: "As stated by Chen (1991), 'the forms of article composition vary depending on the operating approaches designed by the authors.' However, when authors outline their works, they are inevitably and subconsciously affected or dominated by common human principles. Consequently, the completed works, if compared to trees, share fundamental and common trunks despite differing branches." Aesthetic feelings, which are special and complex psychological phenomena, share similar qualities when observed in regularly diverse aesthetic subjects. This similarity is particularly evident when appreciating formal beauty." Only by exploring and understanding the affections and aesthetics that poets intend to convey and identifying the principles governing these phenomena can we comprehend the aesthetic effects of time and space design in poetry. Applying these techniques to design can manifest the value of poetry in cultural creativity. 


\section{Transforming the Time and Space Forms of Poetry into Design}

In this section, we referenced the time and space forms of poetry developed by Huang (1976) and $\mathrm{Li}$ (2007), adopting the following categories: time/space reflection, time/space separation, time/space sympathy, and time/space integration. We analyzed actual design cases to investigate the process of logical deduction and the specific steps used to logically transform poetry to design. The results are detailed in the following subsections (Yeh, 2014).

\subsection{Time/Space Reflection}

"Amid complex temporal and spatial relationships, temporal changes may exist in poems that contain only spatial descriptions in the literal texts, because temporal changes are implied in the spatial variations. Similarly, spatial changes may exist in poems that contain only temporal descriptions in the literal texts, because spatial changes are implied in the temporal variations. This conversion between explicit and implicit ideas is called time/space reflection." Huang (1976) This can be divided into two approaches: expressing time through spatial variations and expressing space through temporal variations. A Message, a poem by Zhang Bi provides an example of time/space reflection: "I go in a dream to the house of Xie; through a zigzag porch with arching rails; to a court where the spring moon lights forever; phantom flowers and a single figure." Analyzing the temporal and spatial structures of the poem shows that the words describe only spaces. However, the spatial transitions from "the house of Xie" to "porch" and "court" indicate the differing times progressing through and waking from a dream. Thus, the actual temporal and spatial transitions are expressed. A corresponding composition design should reflect the transition between the explicit and implicit elements and the form relation in reflection. The visual context of the design should be based on the meanings of crucial words such as "a zigzag porch with arching rails" and "flowers," and color and composition should be designed accordingly. The overall design should focus on realizing the conversion, contrast, symmetry, and reflection between explicit and implicit meanings (Fig. 1).

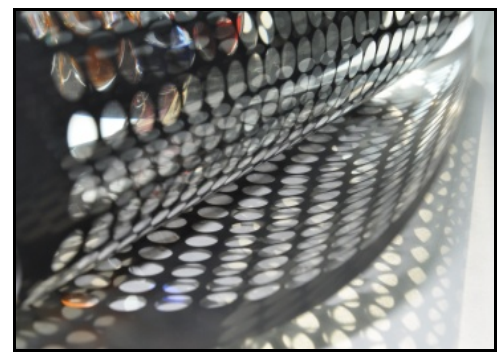

Fig. 1. A case of time/space reflection

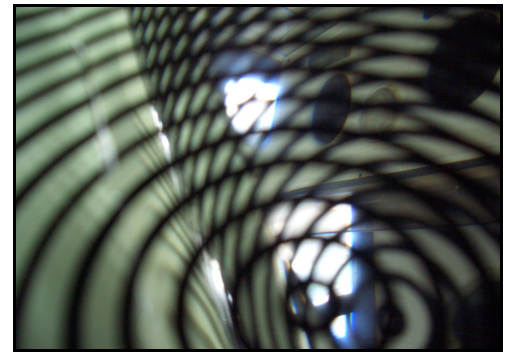

Fig. 2. A case of time/space separation 


\subsection{Time/Space Separation}

"In a poem, time and space coexist as mutual hosts and guests." Time/space separation refers to the alternate expressions (at the verse or couplet level) of time and space that are separated from, yet reflected in each other. In jueju and lushi, two categories of classical Chinese poems, spatial scenes are often used to express vigor, whereas temporal virtual images are used to represent harmony and subtlety. The separation of and contrast between time and space can connect the virtual and physical environment and dynamic and static situations, complementing both types of scenes. In his poem The Tai Town, Wei Zhuang wrote, "The grass by the river grows evenly in the fine rain; Six dynasties are like a dream and birds cry in vain. Nothing is more heartless than the willows of Tai Town; Still veiling the ten-mile-dike just like a misty gown." Analyzing the temporal and spatial structures and patterns of the poem shows that the first verse is a spatial description, the second is a temporal description, and the third and fourth verses return to spatial descriptions. This alternation indicates explicit and contrasting arrangements of time and space. Converting the visual compositions to design requires using structured paragraphs. Based on keywords, such as "fine rain," designers can use ripple shapes to represent the incessant rain. Additional keywords, such as "dream" and "mist" can be used as the basis for the visual design and soft and dreamy materials and colors can be employed. The overall design should focus on recreating the circular and gradually changing relations implied in the poem (Fig. 2).

\subsection{Time/Space Sympathy}

"Sometimes, time and space blend and intertwine in poetry or act as interchangeable exterior and interior elements. The interactions between temporal and spatial elements are dynamic and flexible. Differentiating between time and space is difficult in particular poems. The blending of time and space often creates an impression of lingering emotions, and this type of intricate illusion is called time/space sympathy." In his poem An Autumn Night, Du Mu wrote, "A candle flame flickers against a dull painted screen on a cool autumn night; she holds a small silk fan to flap away dashing fireflies. Above her hang celestial bodies as frigid as deep water; she sat there watching Altair of Aquila and Vega of Lyra pining for each other in the sky." Analyzing the spatial and temporal structures and patterns of the poem shows that although all four verses appear to focus on spatial scenes, the theme of "an autumn night" is implied throughout the verses. Specifically, in the first verse, "candle flame" and "flicker" introduce the concept of an autumn night; in the third verse, an autumn night is implied in "as frigid as deep water;" and the "Altair of Aquila and Vega of Lyra" in the fourth verse again contain references to an autumn night. Therefore, the spatial and temporal elements in this poem blend, serving as simultaneous explicit and implicit meanings in temporal and spatial dimensions; thus, converting the visual elements to design requires expressing complex, illusive, and chaotic, yet orderly dynamics. The converted visual design should be based on keywords such as "a small silk fan" and "painting screen" to express the dynamics of materials. Furthermore, the overall design should focus on expressing the relations among the interchangeable explicit and implicit meanings and balanced proportions (Fig. 3). 


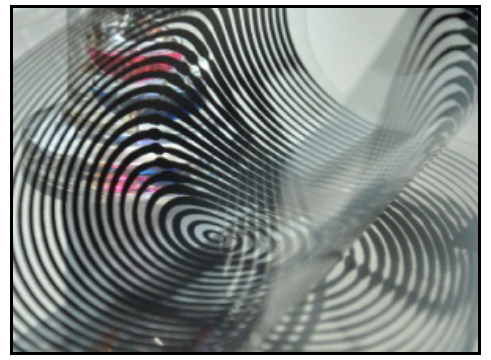

Fig. 3. A case of time/Space sympathy

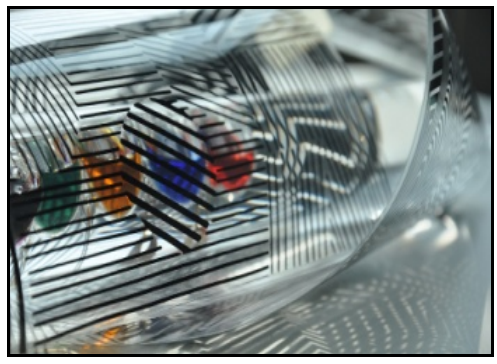

Fig. 4. A case of time/space integration

\subsection{Time/Space Integration}

"Time and space are continuous in the real world; however, they can be divided and overlapped in a poem. Past, present, and future spaces can be reorganized using a new order. This reorganization is called time/space integration." In An Ascent, Du Fu, a Tang poet, wrote, "All over such a vast expanse, the rustling leaves off branches dance. The Yangtze River rises yon, and passes raging on and on. Apart from home so far and long, with autumn, myriad sorrows throng. With illness all my life to fight, I now alone ascend this height." Analyzing the spatial and temporal structures and patterns of the poem shows that the first two verses describe the external scenery the poet saw during a climb, and the following two verses were intended to express the internal self-pity and sorrow the poet experienced during the climb. In this poem, Du $\mathrm{Fu}$ created multiple overlapping and continuous artistic conceptions. The poetic ambience created by this overlap and integration is unmatchable by ordinary poems. Thus, converting the visual compositions to design requires reorganizing diverse elements. The conversion to a visual design should be based on keywords such as "off branches" and "the Yangtze River" to express the dynamics of these features. The overall design should focus on expressing the relations among the overlapping, interacting, circulating, and harmonious movements (Fig. 4).

\section{A Model for Transforming the Time and Space Forms of Poetry to Design}

After conducting case analyses of the four aforementioned types of designs, we examined the following steps for transformation: identifying the origin and background of a poem, analyzing the theme of a poem, exploring the meaningful structure of a poem, converting the time and space forms of a poem to design, determining the expressional properties of the design, and finally completing a design based on the time and space forms of a poem (Table1). The proposed model for converting the time and space forms of poetry to design is detailed in the following sections. 


\subsection{Identifying the Origin and Background of a Poem}

The background and theme of a poem affects the directions of content development and design conversion. This background can be understood by examining external cultural and environmental elements, such as the formation of thoughts, changes in literary materials, effects of religious factors, appreciation of regional scenery, expansion of poetic skills, and the psychology of the poet, and internal individual factors such as family traditions, habits, and inspirations from previous scholars (Lee, 2003). The essence depicted in poems and subjective emotions and ideas of poets are reflected by the following: the pride and ideals hidden in the subconscious, frustrations resulting from obstacles and being unappreciated, sarcastic criticisms regarding absurd political or religious superstitions, concerns regarding national destiny and emperors, the interactions and conflicts between the ideals of pursuing benefits to the family and country versus achieving inner tranquility, the spiritual freedom and liberation that poets pursue in old age, and the dedication to achieving Zen Buddhist states (Liu, 2007). We suggest that the backgrounds and creative motivations of poems are derived from the spiritual awareness, life philosophy, and realizations of the poet. These aspects can be converted to creative design concepts during the brainstorming stage of the creative process and employed to facilitate designing emotional communications.

\subsection{Selecting the Themes of Poems}

It is possible to examine the connotations of the temporal and spatial design, visual context representation, and dynamic temporal and spatial variations in poems. Appropriate poetic contents can be selected based on the anticipated categories and properties of the design. Furthermore, the signifier of reality should be considered.

\subsection{Analyzing the Temporal and Spatial Structures and Patterns of Poems}

Appropriate connotations and implications can be selected from poems after analyzing the composition; this encompasses the external verbal presentations and hidden moral contents of poems. The structures and patterns can be analyzed to explore the sequential levels and structural composition of the poetic conceptions. Visual contexts are examined to determine the presented elements of movement, color, material, and shape. Based on these properties, we divided poems into four time/space categories: reflection, separation, sympathy, and integration.

\subsection{Converting the Time and Space Forms of Poetry to Design}

The artistic conceptions and keywords of poems are converted to various elements, such as shapes, materials, and colors, in actual designs. Specifically, poems featuring time/space reflection focus on expressing the relations among interchangeable exterior and interior positions, contrast, symmetry, and reflection. Poems featuring time/space 
separation are characterized by well-structured paragraphs and circular and evolving relationships. Poems featuring time/space sympathy are often intended to express integrated explicit and implicit meanings, complex illusions, chaos with implicit order, and balanced and harmonious proportions. Poems featuring time/space integration emphasize diverse reorganization, movement, and harmony.

\subsection{Cases of Poetry-Inspired Design}

These cases show the proposed model for converting the time and space forms of poetry to design; we designed this model after examining several design cases, and it should serve as a reference during the execution stages in creative cultural design.

\section{Conclusion}

Li (2007) stated: "Amid the infinity of time and the boundlessness of the universe, thoughts enable people to transcend thousands of years and visions enable people to travel thousands of miles. Poets can roam through the eternity of time and space." Poems represent endless life, and "emotions live on to eternity. An emotion represents imagery, and imagery represents a spiritual realm. In other words, emotions are derived from the scene observed and vice versa" (Zhu, 2003). People are motivated by the sentiments caused by deaths, births, separation, the frustrations of long-distance relationships, and the sorrow resulting from the awareness of the finiteness of human life; thus, people actively seek the elements of timelessness that poets and scholars have described in their writings throughout human history. Dialogs conducted across time and space enable adapting classical Chinese poetry to contemporary and novel images. In this study, we developed a model for converting the time and space forms of poetry to design. Specifically, the emotional demands underlying contemporary design and the beauty of poetry were used as the approaches to creating designs. We also explored the feasibility of applying the cultural connotations of classical poetry to contemporary design.

In recent years, the Taiwanese government has dedicated itself to promoting cultural and creative industries, primarily focusing on industrial applications that involve converting traditional cultural relics into creative designs; however, Chinese cultural relics are diverse, exhibiting distinctive characteristics. Analyzing the connotations and formal changes in the classical poetry culture indicates that the dimensions of poetry differ substantially. In addition, the narratives in poems vary immensely. When analyzing the structural implications of poems, such as color, visual images, temporal and spatial artistic conceptions, and aesthetic expressions, it is worthwhile to discuss methods of converting these forms to designs that apply cultural creativity. This study involved converting poetic elements to designs to allow people to experience and appreciate the ancient beauty of language, enabling traditional culture to regain its vigor. 


\section{References}

1. Cassirer, E.: An essay on man: An introduction to a philosophy of human culture. Yale University Press, London (1962); (Original work published in 1944)

2. Chou, H.P.: The Aesthetics of the Temporal and Spatial Design in Classical Poetry. Wen Chin Publishing Co., Ltd., Taipei (2002)

3. Chen, M.M.: A Collection of Essays on Chinese Teaching. The World of Chinese Language and Literature, Taipei (1991)

4. Xun, F.: Shanjingju Hualun. Yee Wen Publishing Company, Taipei (1971)

5. Huang, Y.W.: Chinese Poetics: Design. Chuliu Publisher, Taipei (1976)

6. Langer, S.K.: Feeling and form: A theory of art developed from philosophy in a new key. Charles Scribner's Sons, NY (1953)

7. Lee, B.R.: The Aesthetics of Du Fu's Poems Inscribed on Paintings. Unpublished Master's Thesis. Master's Program, Department of Chinese, National Taiwan Normal University, Taipei (2003)

8. Liu, M.C.: Explore the Aesthetics Hidden in the Scenery Poems of Xie Lingyun. Unpublished Master's Thesis. Graduate Program, Department of Chinese Literature, National Cheng Kung University, Tainan (2007)

9. Lin, R.-T.: Transforming Taiwan Aboriginal Cultural Features into Modern Product Design: A Case Study of a Cross-cultural Product Design Model. International Journal of Design 1(2), 45-53 (2007)

10. Li, Y.L.: Aesthetics of Poetry. Tung Da Publishing, Taipei (2007)

11. Li, Y.Y.: Culture and Self-Cultivation. Youth Culture Publishing Company, Taipei (1996)

12. Rapaille, C.: The Culture Code. Broadway Books, New York (2006)

13. Worringer, W.: Abstraction and empathy: A contribution to the psychology of style. Ivan R. Dee, Publisher, Chicago (2007)

14. Yeh, M.L.: Application of Poetry in Creative Design. Unpublished doctoral dissertation, National Taiwan University of Arts, Taipei (2014)

15. Zhu, G.Q.: Psychology of Arts. Jin Feng Publishing, Taipei (1988)

16. Zhu, G.Q.: On Beauty. Morning Star Publishing Company, Taipei (2003) 